mostly grade II $(41 ; 60.3 \%)$ and grade III $(20 ; 29.4 \%)$. Of the total number of children with a prenatal diagnosis of hydronephrosis, 28,6\% had VUR.

In the group of children with first febrile UTI, VUR was discovered in $30(36,6 \%)$. With regard to VUR, no significant difference was found between normal pathologic ultrasound findings $(38.1 \%$ vs. $41.7 \%$; p 0.739$)$. No difference was found regarding severity of UTI: urine Leukocyte $<20$ vs. urine Leukocyte $>20(40.3 \%: 43.7 \% ; \mathrm{p}=0,787)$, $\mathrm{CRP}<50$ vs. CRP > $50(37.5 \%: 35.5 \% ; \mathrm{p}=0,46)$ as well as between age of children $<1$ year vs. $>1$ year $(34,5 \%: 41,3 \% ; p=0,141$. Significant differences were found between girls and boys $(\mathrm{M}$ vs.F $=19.0 \%$ vs.34.6\%; $\mathrm{p}=0.0466)$.

Analyzed data point to a higher likelihood of background VUR in recurrent UTI; whereas gender, age, urinary tract ultrasound examination, severity, and other causative agents of UTI have no predictive value for VUR detection. ceVUS is one of the best choices in modern nephrology for the detection of VUR with high sensitivity and negligible side effects.

\section{A RARE CASE OF POLYCYSTIC KIDNEY DISEASE AND MULTICYSTIC DYSPLASTIC KIDNEY IN A PEDIATRIC PATIENT}

Kovačević Ana*, Roić Goran, Trutin Ivana, Valent Morić Bernardica. Sestre milosrdnice University Hospital Centre

\subsection{6/archdischild-2021-europaediatrics.357}

Introduction Cystic kidney disease (CyKD) is one of the most important causes of chronic kidney disease (CKD) in children. Multiple kidney cysts can occur unilaterally (e.g. multicystic dysplastic kidney - MCDK) or bilaterally (e.g. autosomal dominant or autosomal recessive polycystic kidney disease) due to genetic or non-genetic (developmental or acquired) disorders. Here we present a rare case of combined polycystic kidney disease (PCKD) and MCDK in a pediatric patient.

Case Report The patient was admitted to our hospital for the first time at the age of two. She was normally developed (weight: $10.8 \mathrm{~kg}-19$ th percentile, height: $90 \mathrm{~cm}-80$ th percentile) with blood pressure (BP) within normal limits.

Estimated glomerular filtration rate (eGFR) at that time was $70.2 \mathrm{~mL} / \mathrm{min}$ with elevated albumin/creatinine ratio 5,2 $\mathrm{mg} / \mathrm{mmol}$ and she was diagnosed with G2A2 stage of CKD. During her prenatal period oligohydramnios and polycystic kidneys were detected. At birth a palpable mass of the left abdomen was found. First sonography after birth showed hydronephrosis of the right kidney and multiple cysts on the left kidney. Micturating cystourethrography was negative for vesicoureteral reflux. Initial magnetic resonance imaging (MRI) showed enlargement of both kidneys with multiple cysts of various sizes on the left kidney and lesser number of diffuse renal cysts on the right kidney. Cysts weren't found neither on the brain nor on the other abdominal organs. Echocardiography was normal. Family history was negative for kidney diseases. The abdominal and kidney ultrasound of both parents and older brother was normal. At the age of two dynamic scintigraphy with Tc-99m revealed nonfunctional left kidney leading to diagnosis of MCDK.
Follow-up ultrasound showed progressive involution of the MCDK left and multiple very small cysts that generate abnormal parenchymal echogenicity (eg, salt-and-pepper sign) on the right kidney. Functional MRI urography showed nonfunctional MCDK left and functional right kidney with lesser number of small cysts. At the age of four arterial hypertension $(130 / 70 \mathrm{mmHg})$ was diagnosed and ACE inhibitor was introduced. There were no signs of liver disease. Abdominal ultrasound revealed no cysts of liver, spleen or pancreas. Gynecological ultrasound and ophtalmological examination were also normal. Due to parental disapproval, genetic testing wasn't performed.

Conclusion We conclude that, although rare, different types of CyKD can be associated and we should consider it when setting the diagnosis. Due to vast differential diagnosis and overlapping clinical presentations of CyKD genetic testing should be performed whenever possible.

\section{RECURRENT MULTI DRUG RESISTANT URINARY TRACT INFECTIONS IN A THREE-YEAR-OLD HOSPITALIZED CHILD WITH HYPOTONIC CEREBRAL PALSY}

Isac Raluca*, A Blescun, Cl Olariu, R Stroescu, M Gafencu, F Horhat, L Chisavu, G Doros. University of medicine and Pharmacy 'Victor Babes' Timisoara, Department of Pediatrics

\subsection{6/archdischild-2021-europaediatrics.358}

Background Catheter associated urinary tract infection (CAUTI) is a common device-acquired infection and represent a potentially harmful reservoir of resistant uropathogens. Guidelines recommend limitation of catheter use, aseptic catheter insertion, sterile equipment, strict hand hygiene, use of smallest catheter possible and maintenance of a closed drainage system.

Klebsiella Pneumoniae is a non-mobile aerobic rod causing a large spectrum of hospital-acquired infections, especially pneumonia or urinary tract infections (UTI), developing intrinsic resistance genes. Treating multi drug resistant(MDR) gram negative pathogens becomes a challenge for the caregiver.

Vesicoureteral reflux(VUR) consists of backflow of urine from the bladder into the ureters. It can be primary or secondary due to abnormal lower urinary tract function and elevated intravesical pressure. Post void residual(PVR) is a hallmark of detrusor underactivity(DUA) in children.

Case Presentation Summary We present the case a three years old boy, hospitalized for viral encephalitis, undergoing artificial respiratory support and urine catheterization for 6 weeks. Neurological status was hypotonic cerebral palsy and secondary urine incontinence in a previously toilet trained child.

First febrile UTI developed two days after removing urine catheter. High resistant Klebsiella pn. (ESBL+, AAC(3)-II) was treated with a ten-day course of Cephtriaxone and Amikacin. Clinical response to treatment was good with sterile urine culture after 96 hours. After treatment, the child had asymptomatic bacteriuria with MDR Klebsiella pn. in spite of rigorous local hygiene, proper hydration and oral Fosfomycin. Second febrile UTI was accompanied by febrile seizures, and urine culture was positive with MDR Klebsiella (+ESBL or +HL AmpC, Carbapenem impermeability). Treatment with high dose Meropenem (40mg/kg/dose) for twelve days was successful. Third febrile UTI occurred five days after finishing treatment with same MDR Klebsiella strain. Once again, fourteenday high dose Meropenem course was successful. Fourth 